\title{
P50 sensory gating is related to performance on select tasks of cognitive inhibition
}

\author{
CARLY A. YAdON \\ Colorado State University, Fort Collins, Colorado \\ JULIE M. BugG \\ Washington University, St. Louis, Missouri \\ Michael A. Kisley \\ University of Colorado, Colorado Springs, Colorado \\ AND \\ Deana B. Davalos \\ Colorado State University, Fort Collins, Colorado
}

\begin{abstract}
P50 suppression deficits have been documented in clinical and nonclinical populations, but the behavioral correlates of impaired auditory sensory gating remain poorly understood. In the present study, we examined the relationship between P50 gating and healthy adults' performance on cognitive inhibition tasks. On the basis of load theory (Lavie, Hirst, de Fockert, \& Viding, 2004), we predicted that a high perceptual load, a possible consequence of poor auditory P50 sensory gating, would have differential (i.e., positive vs. negative) effects on performance of cognitive inhibition tasks. A dissociation was observed such that P50 gating was negatively related to interference resolution on a Stroop task and positively related to response inhibition on a go/no-go task. Our findings support the idea that a high perceptual load may be beneficial to Stroop performance because of the reduced processing of distractors but detrimental to performance on the go/no-go task because of interference with stimulus discrimination.
\end{abstract}

Broadly defined, sensory gating refers to the brain's selective processing of sensory stimuli. One such modulatory function is the filtering of repetitive stimuli from the environment. Auditory P50 sensory gating is the brain's suppression of an evoked response to a brief auditory stimulus presented just after an identical stimulus. ${ }^{1}$ This ERP appears relatively early in the processing stream (about $50 \mathrm{msec}$ ) and has been suggested to represent an adaptive mechanism that prevents organisms from becoming overwhelmed with redundant sensory information from the environment (Croft, Lee, Bertolot, \& Gruzelier, 2001). However, the functional relevance of this neurophysiological measure has not been demonstrated.

P50 gating is typically quantified by computing a ratio of evoked amplitudes to auditory clicks in a paired-click design (de Wilde, Bour, Dingemans, Koelman, \& Linszen, 2007). Although the auditory modality is by far the most well represented in the literature, there have been attempts to quantify visual (Adler, Waldo, \& Freedman, 1985) and tactile (Arnfred, Eder, Hemmingsen, Glenthøj, \& Chen, 2001) gating. Impairment of auditory P50 gating has been observed in a variety of clinical populations, including individuals with traumatic brain injury (Arciniegas et al.,
2000), Alzheimer's disease (Jessen et al., 2001), panic disorder (Ghisolfi et al., 2006), and Huntington's disease (Uc, Skinner, Rodnitzky, \& Garcia-Rill, 2003). The most notable is schizophrenia (Olincy et al., 2000), for which P50 suppression has been investigated as a potential endophenotype (Freedman et al., 1997; Gottesman \& Gould, 2003). Disrupted P50 gating is not, however, limited to clinical populations. Individual differences in P50 gating have been demonstrated in healthy adults, with some participants' gating scores falling within the range of those observed in individuals with schizophrenia (Patterson et al., 2008). Relatively little is known, however, about the functional consequences of poor sensory gating. As was noted by Potter, Summerfelt, Gold, and Buchanan (2006), especially lacking are data relating P50 gating to measures of cognitive functioning.

In the few published studies in which this topic was examined, the primary cognitive process that has been evaluated is attention. Two approaches have been utilized. One approach involves instructing participants to count clicks that have either a high or low pitch and examining whether attention to the clicks modulates gating. Some findings indicate that gating is attenuated by this manipulation

C. A.Yadon, cyadon@lamar.colostate.edu 
in normal controls, suggesting that attentional goals can influence the effectiveness of sensory gating processes (Guterman \& Josiassen, 1994; Guterman, Josiassen, \& Bashore, 1992; but see Jerger, Biggins, \& Fein, 1992). The second approach involves examining the relationship between performance on neuropsychological measures of attention and P50 sensory gating. For example, in one study, individuals with schizophrenia were divided into low and high P50 abnormality groups on the basis of their P50 sensory gating scores (Erwin, Turetsky, Moberg, Gur, \& Gur, 1998). Those in the high abnormality group performed significantly worse on a distraction measure from the Gordon continuous performance task and on the Trails B shifting and sequencing task. Trends $(p s=.06)$ were observed in the same direction for a vigilance measure from the continuous performance task and the digit symbol task, a commonly used measure of processing speed (Salthouse, 1996). Cullum et al. (1993) similarly reported a positive relationship between performance on a digit vigilance test and P50 suppression for schizophrenia patients but did not find that P50 suppression was related to processing speed in this group; for control participants, no significant correlations were found between P50 suppression and digit vigilance or digit symbol performance. Together, these findings suggest that at least for patients with schizophrenia, attentional processes such as shifting, distraction resolution, and vigilance may share some underlying mechanisms with sensory gating.

Although these studies provide an initial step in characterizing the relationship between sensory gating and higher order cognitive processes, there are important limitations of this work. For instance, additional research is necessary that examines theoretically relevant cognitive processes other than attention. Furthermore, work involving nonclinical samples seems critical (Persons, 1986), in that medication status and/or other clinical features present in the studied samples of individuals with schizophrenia may have influenced the observed relationships between P50 gating and cognition. To counter these limitations, in the present study, we examined the relationship between P50 gating and a select component of executive functioning - inhibition - in healthy younger adults. Our rationale for focusing on measures of cognitive inhibition, including the Eriksen flanker, go/no-go, negative priming, and Stroop tasks, is discussed next.

\section{Rationale for Investigating the Relationship Between P50 Gating and Cognitive Inhibition}

Cognitive inhibition is a multidimensional construct (Kramer, Humphrey, Larish, Logan, \& Strayer, 1994). Current theories posit that inhibition consists of separable but related processes (e.g., Friedman \& Miyake, 2004). For instance, cognitive inhibition is believed to underlie performance on tasks that require the restriction of attentional access, deletion of no-longer-relevant information from attention and working memory, and restraint over habitual or prepotent response tendencies (e.g., Lustig, Hasher, \& Zacks, 2007). It has been suggested that sensory overload produces downstream effects on cognition (Venables, 1964) and that a failure of the P50 filtering mechanism may lead to perceptual, attentional, or other cognitive difficulties. For instance, healthy individuals with poorer auditory P50 gating are more likely to report feeling overwhelmed or bombarded with auditory stimuli from the environment (Kisley, Noecker, \& Guinther, 2004). Similarly, patients with schizophrenia report being overwhelmed by sensory stimuli from the environment; this may be due to fundamental attentional and inhibitory deficits (McGhie \& Chapman, 1961). Sensory overload may affect cognition, for example, when unfiltered sensory stimuli compete with other stimuli or goals for limited attentional resources (e.g., Desimone \& Duncan, 1995; Làdavas, Petronio, \& Umiltà, 1990). Indeed, if sensory gating is perturbed, the failure to filter sensory information may have fairly broad consequences. For example, autism is also associated with a sensory filtering deficit (Talay-Ongan \& Wood, 2000), and this low-level inhibitory deficit has been suggested to contribute to higher order difficulties, such as social interaction and communication, that characterize this disorder (Heal \& Johnson, 1970).

An additional factor that is suggestive of a relationship between P50 gating and cognitive inhibition, revealed in functional neuroimaging studies, is overlap in the neural regions that appear to subserve both P50 gating and performance on cognitive inhibition tasks. Tasks with an inhibitory component consistently recruit the frontal regions of the brain, with the specific distributions of regions that are recruited being task dependent. Many similar inhibitory tasks have been reported to recruit nonoverlapping brain regions. For example, Nee, Wager, and Jonides (2007) conducted a meta-analysis that included PET and fMRI studies of inhibitory tasks requiring interference resolution, which was operationalized as the need to ignore irrelevant information or to withhold a prepotent response in order to make the correct response. Quantitative summaries were provided for classic tasks, such as the Stroop, go/no-go, and Eriksen flanker tasks. For the Stroop task, most activation was in the left dorsolateral prefrontal cortex (DLPFC) and the medial frontal cortex, including the anterior cingulate cortex (ACC). There was also activity to a lesser degree in the right DLPFC and thalamus. Conversely, for the go/no-go task, the strongest response was in the right DLPFC; this stretched into the inferior frontal gyrus. There was also left DLPFC and ACC activity, but this was less pronounced. For the flanker task, the primary activation was in the right DLPFC. Although not included in this meta-analysis, there have also been reports of frontal lobe activation in negative priming, another task involving inhibition. For example, the medial frontal gyrus; inferior, middle, and superior frontal gyri; precentral gyrus; and precentral gyrus pars opercularis were activated in a Stroop version of a negative priming task, which controlled for the Stroop interference effect (Steel et al., 2001). The inferior frontal gyrus was also activated in a visuospatial negative priming task in addition to orbitofrontal regions (Wright et al., 2006).

As in the cognitive inhibition tasks, there is increasingly more evidence to suggest frontal cortex involvement in P50 sensory gating (Grunwald et al., 2003; Korzyukov et al., 2007; Kurthen et al., 2007; Weisser et al., 2001). 
Both fMRI (Marlow-O'Conner, 2005; Tregellas et al., 2007) and lesion (Knight, Staines, Swick, \& Chao, 1999) paradigms have provided evidence for bilateral recruitment of DLPFC in gating. Premotor/supplementary motor cortex (Brodmann's area [BA] 6) and the ACC have also been implicated (Grunwald et al., 2003). In addition, nonfrontal generators in P50 gating are commonly reported. For example, animal models and human studies have yielded strong support for the role of hippocampal (Arciniegas et al., 2001; Bickford-Wimer et al., 1990) and temporal cortex (Korzyukov et al., 2007; Kurthen et al., 2007; Weisser et al., 2001) generators. In light of this, gating has been described as a multistep process, with the frontal cortex potentially regulating or modulating sensory gating by providing additional stimulus evaluation (Grunwald et al., 2003; Weisser et al., 2001).

In addition to the evidence outlined above, one other set of findings motivated our exploration of the relationship between P50 auditory sensory gating and cognitive inhibition. Individuals with schizophrenia, a group for which auditory P50 sensory gating is impaired, perform more poorly than controls on a variety of cognitive inhibition tasks. For example, in this group, inhibitory deficits have been observed on the Stroop (1935) color-naming task (Cohen, Barch, Carter, \& Servan-Schreiber, 1999; Everett, Laplante, \& Thomas, 1989), the attentional network task (a modified version of the Eriksen flanker task; Gooding, Braun, \& Studer, 2006), and the go/no-go and stop signal tasks (Weisbrod, Kiefer, Marzinzik, \& Spitzer, 2000). A common mechanism may underlie these impairments, although this remains speculative, given that the measures of P50 gating were not employed alongside the cognitive inhibition measures in these studies.

\section{A Potential Mechanism Underlying the Putative Relationship Between Auditory P50 Sensory Gating and Cognitive Inhibition}

Above, we provided a few examples of possible negative consequences associated with poor sensory gating. In this section, compelled by load theory (Lavie, Hirst, de Fockert, \& Viding, 2004), we introduce a potential mechanism that may underlie not only the negative but also the potentially positive consequences of poor sensory gating (for a similar idea related to the costs and benefits of increased distractibility, see Healey, Campbell, \& Hasher, 2008). Load theory differentiates between the attentional consequences associated with a high perceptual versus high working memory load. According to load theory, a high perceptual load tends to reduce the processing of distractors, because resources are less available to perceive them. For example, increased perceptual load at fixation has been shown to decrease interference from distractors in visual perception paradigms (e.g., Lavie, 1995; Schwartz et al., 2005). In contrast, a high working memory load tends to increase distractor processing, because resources are less available to actively inhibit them. By this account, if poor sensory gaters' cognitive systems tend to be loaded with extraneous perceptual information relative to good sensory gaters, poor sensory gaters may afford less processing to distracting information and therefore perform better on select tasks. Tests of this prediction feature tasks in which the distractors are concurrently presented along with the perceptual target and therefore compete directly (interfere) with selection of the targets. Tasks that feature these types of stimuli include the negative priming and conflict resolution tasks (Stroop and Eriksen flanker) used in the present study.

Not all of the inhibition tasks used in the present study, however, feature perceptual distractors that are presented in the same visual display as the targets. For example, in the present study, we incorporate a response inhibition task: the go/no-go task. In this paradigm, participants must discriminate between simple stimuli (e.g., discriminate " $f$ " from " $k$ ") that are presented one at a time and quickly emit or withhold a motor response on the basis of a set rule. If visual discrimination is made more difficult, for example because of a high perceptual load (see, e.g., Barnhardt, Ritter, \& Gomes, 2008), responses to no-go stimuli may be inappropriately initiated and sometimes fail to be withheld.

\section{The Present Study: Goals and Predictions}

The goal of the present study was to investigate the relationship between auditory P50 sensory gating and cognitive inhibition using four inhibitory tasks. Drawing from the very limited prior literature on this topic, we anticipated that cognitive inhibition would be related to auditory P50 sensory gating in our sample of healthy young adults. On the basis of load theory, we predicted that the precise nature of this relationship would depend on the type of inhibitory task employed. For the Stroop and Eriksen flanker tasks (conflict resolution tasks), we predicted that poor sensory gating would be associated with reduced interference effects. That is, the high perceptual load associated with poor gating would benefit filtering of distractors in these tasks. For the negative priming task, we examined the degree to which selection of a target was slowed by prior inhibition of the target (i.e., negative priming). To the extent that a high perceptual load reduces processing of distractors, including the active inhibition of them, we predicted that negative priming would be less robust for poor sensory gaters. Finally, for the go/no-go task, we expected the high perceptual load associated with poor sensory gating to interfere with visual discrimination processes that were necessary for distinguishing between targets (go trials) and nontargets (no-go trials) and accordingly, successful stopping performance on no-go trials. As such, we predicted poor sensory gating to be associated with poor response inhibition on this task. Our predictions follow from load theory and are therefore consistent with the notion that poor sensory gating may have both positive and negative downstream effects on the perceptual and cognitive processes involved in cognitive inhibition paradigms.

\section{METHOD}

\section{Participants}

Thirty-four healthy adults ( 8 male; mean age $=20.9$ years, age range $=18-25$ years) receiving credit from an introductory psychology course volunteered to participate in the study. The participants were screened for abnormal hearing, any prior traumatic brain injury 
(Arciniegas et al., 2000; 2 participants were excluded for this), any history of a serious neurological condition, psychiatric condition (bipolar disorder, psychotic disorders), or psychoactive drug use that has been found to affect P50 gating (Nagamoto et al., 1996). We also screened for smoking within $1 \mathrm{~h}$ of the study (Adler, Hoffer, Griffith, Waldo, \& Freedman, 1992). Screening was conducted using a self-report format on a demographic questionnaire. Seven additional participants were excluded for reasons discussed below. The final sample consisted of 25 participants ( 4 male; mean age $=20.9$ years, age range $=18-25$ years).

\section{Design and Procedure}

The research session lasted approximately $3 \mathrm{~h}$ and consisted of two procedures - an EEG and cognitive testing - with the order of these procedures counterbalanced across participants. Prior to engagement in the EEG and cognitive testing, the participants provided informed consent and completed the demographics questionnaire.

\section{Electrophysiological Procedure}

Recording. Electrophysiological data were collected in a soundattenuated room adjacent to the control room. The participants were seated upright in a chair and remained upright during the recording. A brief document was provided that explained the nature of EEG recording, tips for reducing artifacts, and an overview of the task. The participants were encouraged to reduce blinks when possible, especially during the click presentation.

The participants were fitted with a high-density (128-channel) hydrocel geodesic sensor net (Electrical Geodesics, Inc. [EGI], Eugene, OR) and impedances were adjusted to below $40 \mathrm{k} \Omega$ and maintained below $40 \mathrm{k} \Omega$ throughout the recording. ${ }^{2}$ Electrical signals from the scalp were amplified using a Netamps 200 amplifier (EGI) (Gain $=5,000)$, online filtered between 0.1 and $100 \mathrm{~Hz}$ and sampled at $500 \mathrm{~Hz}$. Digitized data were saved and later manipulated using Netstation software, version 4.1.2 (EGI).

During the EEG recording, paired clicks (5-msec white noise bursts) separated by an interstimulus interval of $500 \mathrm{msec}$ were presented with an intertrial interval of 9 sec (Kisley et al., 2004). The clicks were presented against a silent background over speakers (Harman/Kardon model HK206) located $152 \mathrm{~cm}$ from the participant. Intensity was $85 \mathrm{~dB}$ sound pressure level (SPL), determined by a handheld analog sound meter measured at the ear (Extech Instruments, model 407706). The participants watched a muted movie with closed captions during the click presentation, which contained 170 pairs of clicks.

P50 identification. ${ }^{3}$ Raw electrophysiological data were offline band-pass filtered $(10-75 \mathrm{~Hz})$, segmented $(100 \mathrm{msec}$ before and $300 \mathrm{msec}$ after click onset), artifact detected (a segment was excluded from further analysis if it contained more than 10 poorly functioning electrode channels or if it contained an eye blink or eye movement artifact: threshold $=70 \mu \mathrm{V}$ ), baseline corrected relative to a $100-\mathrm{msec}$ prestimulus baseline, averaged, and re-referenced to electrode 100 (TP10/right mastoid) (Luu \& Ferree, 2005). If artifact rejection resulted in less than 75 click pairs per participant, those data were excluded $(n=1)$.

All data were measured from electrode $129(\mathrm{Cz})$ (Luu \& Ferree, 2005), because this electrode has been reported to show the most robust P50 suppression (Nagamoto, Adler, Waldo, Griffith, \& Freedman, 1991). The P50 component was defined as the largest positive peak occurring between 45 and $80 \mathrm{msec}$. Each click's amplitude was measured relative to baseline. The data for a participant were excluded from further analysis if the P50 did not fall within the specified latency window $(n=1)$, the peak amplitudes for the two clicks were not within $10 \mathrm{msec}$ of each other $(n=1)$, or there was no clear P50 $(n=4)$. The P50 component is typically quantified using a ratio of the amplitude of the response to the test click $(T)$, or second click, to the amplitude of the response to the conditioning click $(C)$, or first click: $T / C$. The ratio of P50 amplitudes produces a number between 0 and 1 , with smaller numbers representing greater suppression and, thus, better sensory gating. Three participants' test clicks were just below 0 (a negative number), which produced a negative $T / C$ ratio. In these instances, the $T / C$ ratio was rounded to 0 .

\section{Cognitive Testing Procedure}

The participants completed four cognitive tasks in a counterbalanced order. Completion of the cognitive tasks took approximately $60 \mathrm{~min}$. The tasks, described in detail below, are commonly used indices of inhibitory control (Kramer et al., 1994). E-prime (Psychological Software Tools, Pittsburgh, PA) and Superlab Pro (Cedrus Corporation, San Pedro, CA) software were used to control stimulus presentation and data collection.

The Eriksen flanker task. The flanker task measures the participants' ability to prevent distracting information at an irrelevant location from impeding their selection of information at a relevant location (Eriksen \& Eriksen, 1974). The participants were presented with a string of seven arrows in the middle of the screen and asked to respond to the central arrow in the string. The participants were instructed to press the left key on a keyboard if the central arrow pointed left and the adjacent right key if the central arrow pointed right. During compatible trials, the flanking arrows faced the same direction as the central arrow (e.g., $<<<<<<<$ ). During incompatible trials, the flanking arrows faced the opposite direction from the central arrow (e.g., $<<<><<<$ ), requiring the participants to inhibit the response associated with the flankers and to select the response associated with the central arrow. Neutral trials, in which the flankers pointed either up or down, were also included. These trials are considered to be neutral in that up and down arrows were not assigned to a response key.

Twenty-four practice trials were followed by five blocks of 72 trials each. Compatible, incompatible, and neutral trials were randomly presented during the task, with an equal number of each trial type occurring within a block. The stimuli remained onscreen until a response was made. A fixation cross also appeared onscreen, below the central arrow, and remained onscreen during the 1,000-msec intertrial intervals.

Both accuracy and reaction time were recorded. The index of inhibition in this task was a proportional interference score that was derived by subtracting mean neutral trial reaction time from mean incompatible trial reaction time, then dividing the obtained value by the mean neutral trial reaction time (see, e.g., Brink \& McDowd, 1999). This measure accounts for baseline differences in processing speed. One participant's data were unusable for this task; the error rate was $96 \%$ (more than $17 \mathrm{SD}$ s from the mean error rate) for incongruent trials, suggesting that this participant's performance was not according to task instructions.

The go/no-go task. The go/no-go task measures inhibition of a prepotent or habitual motor response (e.g., Donders, 1969). This task included two phases. During the initial go phase, the participants were asked to press a designated response key on the keyboard as quickly as possible each time a letter was presented. The go phase consisted of 6 practice trials and 50 task trials, presented at a rate of 1 letter every 1,400 msec. Six lowercase letters were used as stimuli ("c," "f," "k," "l," "n," and "v"). During the no-go phase, the participants were instructed to press the same key that was used during the go phase each time any letter was shown except the letter "f." When "f" appeared, the participants had to withhold (inhibit) the habitual keypress and wait for the next stimulus to appear. The no-go phase consisted of three blocks of 50 trials. Of the 150 trials, $30(20 \%)$ were randomly appearing no-go trials containing the letter "f." The remaining trials consisted of an equal number of go trials containing the other five letters.

To measure inhibition, stopping error was computed as the percentage of no-go trials during which the participants failed to withhold the keypress responses (i.e., they pressed the key when the stimulus was "f").

The negative priming task. The negative priming task was designed to measure inhibition by assessing the additional time that it takes to respond to stimuli that have recently been inhibited during a selective attention task (Neill, 1977; Tipper, 1985). During the task, 
the participants viewed a prime trial, consisting of one shape that was outlined in red and a second shape that was outlined in green (squares, circles, triangles, or diamonds). The prime trial was presented briefly $(250 \mathrm{msec})$ before being replaced by a probe $(250-\mathrm{msec})$ trial $1,000 \mathrm{msec}$ later. The probe trial consisted of one shape outlined in red and a second shape outlined in green. Following the probe trial, the participants were asked to press the "yes" key on the keyboard if the red shape from the prime trial was the same as the red shape from the probe trial and the "no" key on the keyboard if they differed.

There were three trial types: attend trials, control trials, and ignore trials. On attend trials, the red shapes on the probe and prime trials were the same. On control trials, the red shape from the probe was different from the green or red shape from the prime trial. In contrast, on the ignore trials, the red shape from the probe trial was the same as the green shape from the prime trial. This was the critical condition for evaluating inhibition in this task, because the participants were required to attend to a shape that was previously ignored in the prime trial. More specifically, the measure of inhibition is negative priming, which refers to the degree to which reaction times on the ignore trials are prolonged relative to those on the control trials. To derive this measure, we subtracted average control reaction time from average ignore reaction time. In this case, a larger difference refers to stronger inhibition.

The participants completed two versions of this task back to back with the order counterbalanced across participants. One version contained the attend and ignore trials, and the second contained the attend and control trials. Trial type and left/right placement of target (red) and distractor (green) shapes were randomized within each version. Prior to beginning each version of the task, six practice trials were administered. Two participants' data were excluded from this task: 1 whose negative priming score was close to $4 S D$ from the mean and the other because of computer error.

The Stroop task. The Stroop task measures the participants' ability to inhibit a prepotent but goal-irrelevant response by asking them to name the colors of words that are themselves color words (Stroop, 1935). Stroop interference refers to the additional time it takes to name the colors of the words in an incongruent (e.g., RED in blue) relative to a congruent (e.g., RED in red) or neutral (e.g., CAT in red) condition. Unlike the go/no-go task, the prepotent response (word reading) is not developed during the task itself but is instead developed through the participants' repeated experiences with word reading that they bring to the laboratory. The task began with a response mapping phase, during which the participants were familiarized with the colors (red, green, or blue) that had been assigned to three adjacent response keys on a keyboard. During this phase, asterisks were presented onscreen one at a time in red, green, or blue and the participants pressed the response key that corresponded to
Table 1

Descriptive Statistics for

Electrophysiological and Inhibitory Measures

\begin{tabular}{lrrr}
\hline & $M$ & $S D$ & $N$ \\
\hline P50 $C$ amplitude & 3.32 & 1.89 & 25 \\
P50 $T$ amplitude & 0.98 & 0.84 & 25 \\
P50 $T / C$ ratio & 0.33 & 0.22 & 25 \\
P50 C latency & 65.40 & 3.73 & 25 \\
P50 $T$ latency & 65.40 & 4.22 & 25 \\
Stroop interference & 0.24 & 0.18 & 23 \\
No-go stopping error & 16.20 & 10.39 & 25 \\
Eriksen interference & 0.21 & 0.11 & 24 \\
\hline
\end{tabular}

the color of the asterisk. Thirty-six trials were provided. The task itself consisted of three conditions (108 trials/condition) presented in a fixed-order (blocked) design. The participants were instructed to press the response key that corresponded to the color that the words were printed in, ignoring the word, as quickly as possible without sacrificing accuracy. For the neutral condition, the words NIGHT, DAY, and NOON appeared every $500 \mathrm{msec}$ in red, blue, and green. In the congruent condition, RED, BLUE, and GREEN appeared in colors consistent with the word (e.g., RED in red), whereas RED, BLUE, and GREEN appeared in a color that was inconsistent with the word's content in the incongruent condition.

As in the flanker task, a proportional interference score was used as an index of inhibition. We subtracted the neutral condition reaction time from the incongruent trial reaction time then divided by the neutral condition reaction time. Two participants' data were not usable for this task because of excessive errors: The error rate for 1 participant for incongruent trials was $97 \%$ (more than 7 SD from the mean error rate). The other participant did not produce any correct responses on these trials.

\section{RESULTS}

\section{Group-Level Analysis of ERP Data}

For the P50 component, a dependent $t$ test confirmed that peak latency did not differ for the conditioning (Click 1) and test (Click 2) clicks $[t(24)=-0.090$, n.s.]. Critically, however, the amplitude of the two clicks differed $[t(24)=7.08, p<.001]$. The amplitude of the conditioning click was significantly higher than that of the test click; this demonstrates a group-level sensory gating effect (Table 1, Figure 1).

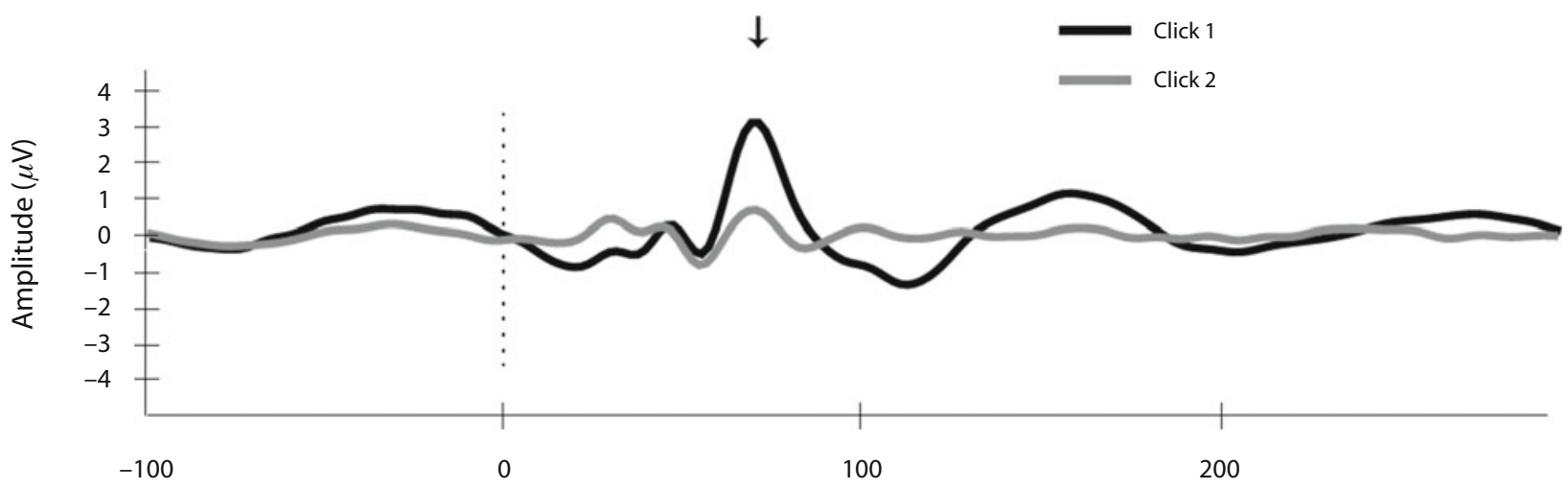

Time (msec)

Figure 1. Grand average P50 (arrow) ERP waveforms presented as amplitude of the two auditory clicks as a function of time. Click 2 (light gray) has significantly reduced amplitude as compared with Click 1 (dark gray) — the typical P50 sensory gating effect. The dotted line indicates stimulus onset. All data are reported from electrode $\mathrm{Cz}, \boldsymbol{n}=\mathbf{2 5}$. 


\section{Cognitive Inhibition Tasks}

To confirm that the typical effects were present for our versions of each cognitive inhibition task, a series of group-level planned comparisons were conducted on the mean reaction time data for each task. First, for the Eriksen flanker task, reaction times were longer on incompatible $(M=560.69 \mathrm{msec})$ than on compatible $(M=444.17 \mathrm{msec})$ $[t(23)=-7.47, p<.001]$ or on neutral $(M=465.84 \mathrm{msec})$ $[t(23)=-8.08, p<.001]$ trials, demonstrating the standard compatibility effect. For the go/no-go task, mean stopping error was $16.20 \%$, and reaction times were longer for go trials during the no-go phase $(M=363.73 \mathrm{msec})$ than during the go phase $(M=276.49 \mathrm{msec})$, reflecting the additional cost of the no-go instruction $[t(24)=-20.49, p<$ $.001]$. For the Stroop task, the standard interference effect was obtained. Response times on incongruent trials $(M=$ $669.23 \mathrm{msec}$ ) were significantly longer than those on congruent $(M=494.39 \mathrm{msec})[t(22)=-8.41, p<.001]$ or neutral $(M=540.38 \mathrm{msec})[t(22)=-6.37, p<.001]$ trials. Finally, for the negative priming task, reaction times were significantly faster for attend $(M=413.84 \mathrm{msec})$ than for control $(M=446.73 \mathrm{msec})[t(24)=2.12, p<.05]$ trials. However, the negative priming effect was not observed, because reaction times were similar for ignore $(M=$ $429.54 \mathrm{msec})$ and control $(M=437.40 \mathrm{msec})[t(24)=$ -0.362 , n.s.] trials. Because we could not replicate the group-level negative priming effect observed in prior reports, we excluded this measure from further analyses.

\section{Interrelationships Among \\ Cognitive Inhibition Tasks}

Previous studies have demonstrated heterogeneity among inhibitory tasks (Kramer et al., 1994); thus, it was not surprising that none of our inhibition measures were significantly correlated with each other (Table 2 ).

\section{P50 and Cognitive Inhibition}

Of primary interest was the relationship between P50 suppression and cognitive inhibition. ${ }^{4}$ We examined this relationship using a two-step approach. First, we conducted a series of correlational analyses to examine the bivariate relationships between each of the three measures of inhibition (described above) and P50 suppression (the ratio of the amplitude of the second click to that of the first click). ${ }^{5}$ Then, to determine whether the dependent correlations differed reliably from one another, we used William's $t$ test to compare the magnitudes of the correlations.

As for the bivariate relationships, the proportional interference score from the Stroop task was negatively cor-

Table 2

Correlations for P50 Suppression and Inhibitory Measures

\begin{tabular}{|c|c|c|c|c|}
\hline & $\begin{array}{c}\text { P50 T/C } \\
\text { Ratio } \\
\end{array}$ & $\begin{array}{c}\text { Stroop } \\
\text { Interference }\end{array}$ & $\begin{array}{c}\text { No-Go } \\
\text { Stopping } \\
\text { Error }\end{array}$ & $\begin{array}{c}\text { Eriksen } \\
\text { Interference }\end{array}$ \\
\hline P50 $T / C$ ratio & - & $-.523^{*}$ & $.452^{*}$ & -.013 \\
\hline Stroop interference & & - & -.338 & -.181 \\
\hline No-go stopping error & & & - & .115 \\
\hline Eriksen interference & & & & - \\
\hline
\end{tabular}
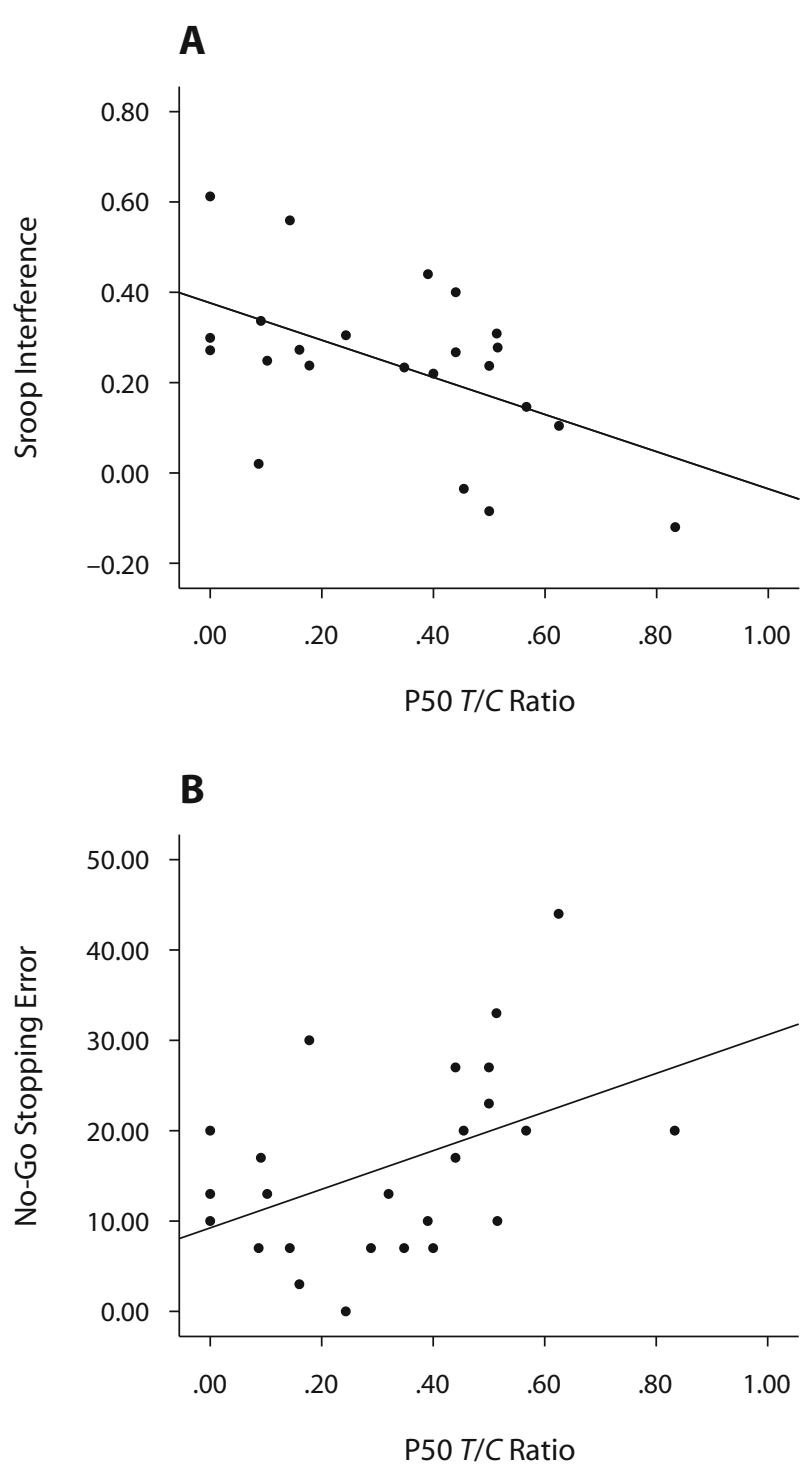

Figure 2. Differential relationships between P50 suppression (abscissas) and cognitive inhibition (ordinates) for (A) Stroop and (B) go/no-go inhibition tasks.

related with P50 suppression $[r(21)=-.523, p=.010]$. This result suggests that the participants who showed reduced interference (i.e., were better at inhibiting the word dimension) on the Stroop task had worse P50 gating (Table 2, Figure 2). The opposite relationship was observed for the inhibitory control measure from the go/no-go task-stopping error. The positive relationship between P50 gating and stopping error during the no-go phase suggests that better response inhibition during the go/no-go task was associated with better P50 gating $[r(23)=.452$, $p=.023]$ (Table 2, Figure 2). ${ }^{6}$ Interference from the Eriksen flanker task $[r(22)=-.013$, n.s. $]$ was not significantly correlated with P50 suppression (Table 2).

As for the comparison of the correlations just described, the William's $t$ test indicated that the relationship between P50 and Stroop interference did not differ from the relationship between P50 and go/no-go stopping error $[t(21)=$ 
0.34, n.s.]. This result suggests that the relationship between Stroop interference and P50 gating did not differ in magnitude from the relationship between go/no-go stopping error and P50 gating. Rather, these correlations, as was reported above, differ only in direction. Two additional William's $t$ tests indicated that the relationship between P50 gating and the interference score from the Eriksen flanker task (see Table 2 for $r$ values) was significantly weaker than the relationship between P50 and Stroop interference $[t(20)=2.07, p<.05]$ and marginally weaker than the relationship between the P50 and go/no-go stopping error $[t(21)=1.68($ critical value $=1.72), p>.05]$. These findings are consistent with the pattern of significant effects for the bivariate correlations reported above.

For our sample, we computed the split-half reliability associated with each cognitive inhibition measure. For the Stroop task, given the blocked design, we calculated the proportional interference score using the first half of the trials in each block and then again using the second half of the trials in each block. The proportional interference score from the first half correlated reliably with the same score from the second half of the Stroop task $[r(21)=.547, p=$ $.007]$. For the Eriksen flanker task, given the mixed-trial design, we split the total trials in half and calculated the proportional interference score for each half. These scores were also reliably correlated $[r(22)=.751, p<.001]$. For the go/no-go task, we calculated the stopping error for the first half of the no-go phase and the second half of the no-go phase. These scores were marginally reliably correlated $[r(23)=.389, p=.054]$. To estimate an average of all possible split-half correlations, we also computed Cronbach's alpha for each cognitive inhibition measure. The resultant values were $.704, .845$, and $.526^{7}$ for the proportional interference Stroop score, the proportional interference Eriksen flanker score, and the stopping error on the go/no-go task, respectively. These results suggest that our cognitive tasks met an acceptable level of reliability.

\section{DISCUSSION}

The purpose of this study was to examine the relationship between measures of cognitive inhibition and auditory P50 sensory gating. As was anticipated, select measures of cognitive inhibition were related to sensory gating in a manner consistent with predictions compelled by load theory. Our primary finding was a dissociation, such that the proportional interference score from the Stroop task was negatively related to P50 sensory gating, whereas stopping error in the go/no-go task was positively related to P50 sensory gating. This pattern suggests that the participants who responded less strongly to the second click than to the first click in the P50 paradigm performed more poorly on the measure of inhibition from the Stroop task, yet showed enhanced performance on the inhibition measure from the go/no-go task. ${ }^{8}$ This is a novel finding with important theoretical and, potentially, clinical implications.

\section{Implications for Load Theory}

At the most basic level, our finding suggests that processes supporting P50 sensory gating overlap with those that support behavioral indices of inhibition. That the proportional interference score from the Eriksen flanker task was not related to P50 sensory gating indicates that the overlapping process is not a general inhibitory process. The differential correlations between P50 gating and performance on the Stroop and go/no-go tasks also support this conclusion and, furthermore, suggest that taskspecific inhibitory processes or other task-specific factors may explain the overlap between P50 sensory gating and these behavioral indices of inhibition.

We posited that poor sensory gating might be associated with a high perceptual load and that a high perceptual load would be associated with different consequences for different types of cognitive inhibition tasks (see Lavie et al., 2004). Our prediction that a high perceptual load would afford reduced attention to distractors for conflict resolution tasks was supported by our finding that poor P50 suppressors had a lower proportional interference score on the Stroop. Our prediction that participants with a high perceptual load would perform more poorly on a response inhibition task that requires visual discrimination of targets from nontargets was also supported. Poor P50 suppressors had greater stopping error on the go/no-go task.

Given that the Eriksen flanker task, like the Stroop task, involves conflict resolution triggered by concurrently presented distracting and target information, we predicted that a high perceptual load would be related to better interference resolution on the flanker task. In contrast to our results for the Stroop (and go/no-go) task(s), our prediction for flanker interference was not supported (we found no relationship between this task and P50 suppression). Given the similar reliability observed for the Stroop and Eriksen flanker tasks in our sample, it is unlikely that the absence of a relationship between P50 and the flanker task relates to the reliability of this measure. However, there are a number of methodological differences between the Stroop and Eriksen flanker tasks that could explain why Stroop interference, but not flanker interference, shared a relationship with gating in the present study. For example, our formats for the two tasks were different. For the blocked format used in the Stroop task, trials of one type were presented together (all the incongruent, congruent, and neutral items were presented in separate respective blocks). For the mixed format used in the Eriksen task, the incongruent, congruent, and neutral trial types were randomly intermixed throughout the task. A blocked format, wherein participants can predict the upcoming trial type, can bias the participants toward use of proactive control modes where selection of relevant information (and suppression of irrelevant information) is maintained tonically across the course of a block (Braver, Gray, \& Burgess, 2007). In contrast, a mixed format, wherein participants cannot accurately predict the upcoming trial type, can bias the participants toward a reactive mode whereby they engage interference resolution or suppression processes in a transient fashion, as needed, trial by trial. Some evidence suggests that perceptual load impacts tonic attentional selection processes (Barnhardt et al., 2008). Thus, our discrepant results for the relationship between the Stroop and Eriksen flanker tasks and P50 gating might relate to the 
blocked versus mixed task formats. That is, the reduced processing afforded to distractors under a high perceptual load may be particularly beneficial in contexts in which the distractors must constantly be inhibited, which was the case for the Stroop but not the Eriksen flanker task.

The discrepant relationship between P50 gating and performance on the Stroop and Eriksen flanker tasks may also be related to modality. Some evidence suggests that the link between sensory processing and performance on cognitive tasks is modality specific, with auditory measures being more closely tied to verbal than to visuospatial tasks (Brumback, Low, Gratton, \& Fabiani, 2004). Auditory sensory gating may relate to performance on the Stroop task but not on the flanker task, given that the former involves verbal distraction, whereas the latter involves spatial distraction.

\section{Alternative Theoretical Accounts}

Attentional load theory provides the most comprehensive account of our findings. There are, however, other explanations that can account for a subset of our data. We review these explanations because the present findings do not eliminate the possibility that other factors, in addition to perceptual load, may underlie the relationship between sensory gating and cognitive inhibition. For example, another explanation for the obtained pattern of less Stroop interference for poor sensory gaters centers on the definition of relevance. Information can be relevant to the degree that the processing of it is important for the current task context. Task instructions generally inform participants about what is relevant. Information can also be relevant to the degree that it is important in everyday contexts. Here, individuals learn what is relevant through repeated experience or learning processes. One might refer to the former as task-specific relevance and the latter as usual or everyday relevance. In the Stroop task, instructions inform the participants that their goal is to name the color of the word stimuli. Thus, color is relevant in a task-specific sense. In contrast, the word dimension is relevant in a usual sense, in that everyday experience with word stimuli involves reading them, perhaps automatically (e.g., Cohen, Dunbar, \& McClelland, 1990; MacLeod \& Dunbar, 1988). Bearing in mind that P50 gating is considered to be an adaptive process, whereby the processing of irrelevant (e.g., redundant or uninformative) stimuli is suppressed, the intriguing possibility exists that good sensory gaters tend to gate on the basis of usual rather than task-specific relevance. As such, one would expect that good sensory gaters are more likely to filter repetitive sounds, which are usually irrelevant, as well as the color of words, which is usually irrelevant, which would lead to greater Stroop interference scores, as was observed here.

Such an explanation would explain the lack of a relationship between P50 gating and proportional interference scores on the Eriksen flanker task, the task that is arguably most computationally similar to a Stroop task. That is, in the flanker task, the relevant stimulus is a central arrow, which is surrounded by flanking arrows. Because everyday life is devoid of experiences that involve processing strings of arrows, there is no preexisting or usual definition of relevance in this paradigm, only task-specific relevance as defined by the instructions. Therefore, if we expect that good sensory gaters filter stimuli on the basis of everyday relevance, the flanker task should not distinguish good from poor P50 sensory gaters. This theoretical framework can explain our results for the Stroop and flanker tasks, but unlike load theory, it cannot adequately explain our findings for the go/no-go task. Recall that good P50 sensory gaters demonstrated better response inhibition by reducing stopping errors in the go/no-go task. As in the flanker and negative priming tasks, there is no preexisting or usual definition of relevance in the go/no-go task. Thus, the idea that good gaters gate what is usually irrelevant, with such a mechanism tending to enhance performance on the no-go trials, seems unlikely.

The differences in the obtained relationships between P50 sensory gating and performance on the three cognitive inhibition tasks may also be related to several nonoverlapping patterns of neural activation that characterize the inhibition tasks. Although, in some instances, fMRI data point to common (overlapping) areas for inhibition tasks (e.g., Nee et al., 2007, reported the strongest activation in the right DLPFC for both a go/no-go task and a flanker task), many researchers have found regionally specific activation patterns for particular types of inhibition tasks. For example, conflict resolution tasks frequently recruit the ACC (Nee et al., 2007), whereas response inhibition tasks also recruit other regions that are related to P50 gating, such as the supplementary motor area (Picton et al., 2007). Response inhibition may be most similar to the low-level sensory inhibition that is evoked in the P50 paired-click paradigm; in fact, these two processes have been linked to a common region of the brain that may facilitate inhibitory processing. More specifically, Picton et al. found that patients with lesions to BA 6 had increased false alarms (what we report as stopping error) on a go/no-go task, and this same area, the premotor/ supplementary motor cortex, has also been implicated in P50 gating (Grunwald et al., 2003). Importantly, BA 6 has been ruled out as a structure exclusively responsible for motor tasks (Picard \& Strick, 2001) and is known to contribute to complex cognitive tasks, such as inhibition. This prefrontal area may contribute broadly to the modulation of sensory and motor-based inhibition processes. Such an explanation would account for the relationship between poor gating and higher stopping error in the present study.

\section{Clinical Implications}

Our finding that P50 gating was significantly, but differentially (in a directional sense, but not in terms of magnitude), related to performance on the Stroop and go/ no-go tasks provides novel information about the behavioral correlates of P50 gating. These correlates are potentially important for expanding our understanding and treatment of schizophrenia, a group for which P50 gating deficits are a potential endophenotype. Before strong conclusions can be drawn, however, further investigation is necessary to determine whether the patterns obtained here are also present in a sample of individuals with schizo- 
phrenia. Some results suggest that the patterns may differ. For example, individuals with schizophrenia, who tend to show deficient gating, fail to attend to the relevant stimulus dimension (i.e., color) on the Stroop task; most commonly in single-trial computerized tasks, such as the one that we administered, an increase in errors (rather than in reaction times) relative to those of controls is observed (Barch, Carter, Hachten, Usher, \& Cohen, 1999). We, in contrast, found that healthy adults who were poor gaters tended to exhibit less Stroop interference than those who were good gaters. More directly, Erwin et al. (1998) observed that individuals with schizophrenia with poor gating scores performed more poorly than those with better gating scores on a distraction measure from the Gordon continuous performance task. One possible explanation for the seemingly discrepant influences of poor sensory gating on susceptibility to distraction in healthy controls versus individuals with schizophrenia may relate to the degree to which nongated information is further processed. Although, for healthy controls, such information may result in a high perceptual load, for individuals with schizophrenia, such information may be attended to the degree that it creates a working memory load. A working memory load has been shown to negatively impact distraction (e.g., interference) control (Lavie et al., 2004).

\section{Limitations and Future Directions}

Generally speaking, methodological factors have been found to have an impact on P50 gating measures (de Wilde et al., 2007). There are several methodological limitations in the present study that should be noted. We did not exclude participants for a family history of psychotic disorders or bipolar disorder. Some findings suggest that first-degree relatives of individuals with schizophrenia also show impaired P50 gating relative to control participants (Clementz, Geyer, \& Braff, 1998). Thus, it is possible that our sample includes a greater proportion of poor gaters than has been reported in some existing studies (e.g., Waldo et al., 1994; but see Patterson et al., 2008, for similar distributions of scores in a college sample). Also, our power to detect some effects, such as the relationship between P50 amplitude difference scores and the cognitive inhibition measures, may have been low because of our relatively small sample size.

The cognitive behavioral tasks for this study were chosen to represent specific types of inhibition; however, they are not necessarily prototypical tasks. For instance, a mixed rather than blocked trial version of the Stroop task may not be related to P50 gating. Similarly, although the P50 measure reflects gating of auditory information, each of the cognitive inhibition tasks entailed visual stimuli, some of which was verbal in nature and some of which was visuospatial. Whether gating of visual stimuli would be more strongly related to performance on tasks such as the Eriksen flanker task (or remain related to the Stroop and go/no-go tasks) remains to be explored. Furthermore, although we used the approach of correlating measures across distinct paradigms, a more precise pinpointing of the processes that overlap between sensory gating and some types of inhibition tasks might be gleaned by using a single paradigm in which sensory load is manipulated and the effects on response inhibition, for example, are measured.

\section{Conclusion}

The present study contributes to the scant literature aimed at characterizing the cognitive correlates of sensory gating processes. In particular, it represents an important first step toward understanding how inhibition of sensory information is related to other forms of inhibition, including response inhibition and interference control. The present findings suggest that a complete model detailing the cognitive correlates of poor sensory gating must include consequences that are detrimental (i.e., poor response inhibition), as well as beneficial (i.e., reduced susceptibility to certain types of interference). Further refinement of such a model will require studies that include tasks with inhibitory components, as well as tasks that rely on other, noninhibitory processes putatively linked to sensory gating. The theoretical notions advanced in this article should provide guidance regarding the nature of these processes and, as such, motivate future work in this area.

\section{AUTHOR NOTE}

The authors thank Aubrey Anthony, Kathleen Kelsey, Lauren Shirley, and Becky Tucker for assistance with data collection and analysis. J.M.B. was supported by National Institute on Aging Grant 5T32AG00030 during writing of the manuscript. Correspondence concerning this article should be addressed to C. A. Yadon, 1876 Campus Delivery, Department of Psychology, Colorado State University, Ft. Collins, CO 80521 (e-mail: cyadon@lamar.colostate.edu).

\section{REFERENCES}

Adler, L. E., Hoffer, L. J., Griffith, J., Waldo, M. C., \& FreedMAN, R. (1992). Normalization by nicotine of deficient auditory sensory gating in the relatives of schizophrenics. Biological Psychiatry, 32, 607-616.

Adler, L. E., Waldo, M. C., \& Freedman, R. (1985). Neurophysiologic studies of sensory gating in schizophrenia: Comparison of auditory and visual responses. Biological Psychiatry, 20, 1284-1296.

Arciniegas, D. B., Olincy, A., Topkoff, J., McRae, K., Cawthra, E., Filley, C. M., ET AL. (2000). Impaired auditory gating and P50 nonsuppression following traumatic brain injury. Journal of Neuropsychiatry \& Clinical Neuroscience, 12, 77-85.

Arciniegas, D. B., Topkoff, J. L., Rojas, D. C., Sheeder, J., Teale, P., YounG, D. A., ET AL. (2001). Reduced hippocampal volume in association with P50 nonsuppression following traumatic brain injury. Journal of Neuropsychiatry \& Clinical Neuroscience, 13, 213-221. doi:10.1176/appi.neuropsych.13.2.213

Arnfred, S. M., Eder, D. N., Hemmingsen, R. P., GlenthøJ, B. Y., \& Chen, A. C. N. (2001). Gating of the vertex somatosensory and auditory evoked potential P50 and the correlation to skin conductance orienting response in healthy men. Psychiatry Research, 101, 221-235.

Barch, D. M., Carter, C. S., Hachten, P. C., Usher, M., \& Cohen, J. D. (1999). The "benefits" of distractibility: Mechanisms underlying increased Stroop effects in schizophrenia. Schizophrenia Bulletin, 25, 749-762.

Barnhardt, J., Ritter, W., \& Gomes, H. (2008). Perceptual load affects spatial and nonspatial visual selection processes: An eventrelated brain potential study. Neuropsychologia, 46, 2071-2078. doi:10.1016/j.neuropsychologia.2008.02.007

Bickford-Wimer, P. C., Nagamoto, H., Johnson, R., Adler, L. E., Egan, M., Rose, G. M., \& Freedman, R. (1990). Auditory sensory gating in hippocampal neurons: A model system in the rat. Biological Psychiatry, 27, 183-192.

Braver, T. S., Gray, J. R., \& Burgess, G. C. (2007). Explaining the 
many varieties of working memory variation: Dual mechanisms of cognitive control. In A. R. A. Conway, C. Jarrold, M. J. Kane, A. Miyake, \& J. N. Towse (Eds.), Variation in working memory (pp. 76-106). New York: Oxford University Press.

BrinK, J. M., \& McDowD, J. M. (1999). Aging and selective attention: An issue of complexity or multiple mechanisms? Journals of Gerontology, 54B, P30-P33.

Brumback, C. R., Low, K. A., Gratton, G., \& Fabiani, M. (2004). Sensory ERPs predict differences in working memory span and fluid intelligence. NeuroReport, 15, 373-376.

Clementz, B. A., Geyer, M. A., \& Braff, D. L. (1998). Poor P50 suppression among schizophrenia patients and their first-degree biological relatives. American Journal of Psychiatry, 155, 1691-1694.

Cohen, J. D., Barch, D. M., Carter, C., \& Servan-Schreiber, D. (1999). Context-processing deficits in schizophrenia: Converging evidence from three theoretically motivated cognitive tasks. Journal of Abnormal Psychology, 108, 120-133.

Cohen, J. D., Dunbar, K., \& McClelland, J. L. (1990). On the control of automatic processes: A parallel distributed processing account of the Stroop effect. Psychological Review, 97, 332-361.

Croft, R. J., Lee, A., Bertolot, J., \& Gruzelier, J. H. (2001). Associations of P50 suppression and desensitization with perceptual and cognitive features of "unreality" in schizotypy. Biological Psychiatry, 50, 441-446.

Cullum, C. M., Harris, J. G., Waldo, M. C., Smernoff, E., Madison, A., Nagamoto, H. T., ET AL. (1993). Neurophysiological and neuropsychological evidence for attentional dysfunction in schizophrenia. Schizophrenia Research, 10, 131-141.

Desimone, R., \& Duncan, J. (1995). Neural mechanisms of selective visual attention. Annual Review of Neuroscience, 18, 193-222.

de Wilde, O. M., Bour, L. J., Dingemans, P. M., Koelman, J. H. T. M., \& Linszen, D. H. (2007). A meta-analysis of P50 studies in patients with schizophrenia and relatives: Differences in methodology between research groups. Schizophrenia Research, 97, 137-151. doi:10.1016/ j.schres.2007.04.028

Donders, F. C. (1969). On the speed of mental processes. In W. G. Koster (Ed. \& Trans.), Attention and performance II (pp. 412-431). Amsterdam: North-Holland. (Original work published 1868)

Eriksen, B. A., \& ERIKSEN, C. W. (1974). Effects of noise letters on the identification of a target letter in a nonsearch task. Perception \& Psychophysics, 16, 143-149.

Erwin, R. J., Turetsky, B. I., Moberg, P., Gur, R. C., \& Gur, R. E. (1998). P50 abnormalities in schizophrenia: Relationship to clinical and neuropsychological indices of attention. Schizophrenia Research, 33, 157-167.

Everett, J., Laplante, L., \& Thomas, J. (1989). The selective attention deficit in schizophrenia: Limited resources or cognitive fatigue? Journal of Nervous \& Mental Disease, 177, 735-738.

Freedman, R., Coon, H., Myles-Worsley, M., Orr-Urtreger, A., OLINCY, A., DAVIS, A., ET AL. (1997). Linkage of a neurophysiological deficit in schizophrenia to a chromosome 15 locus. Proceedings of the National Academy of Sciences, 94, 587-592.

Friedman, N. P., \& MiYaKe, A. (2004). The relations among inhibition and interference control functions: A latent-variable analysis. Journal of Experimental Psychology: General, 133, 101-135.

Fuerst, D. R., Gallinat, J., \& Boutros, N. N. (2007). Range of sensory gating values and test-retest reliability in normal subjects. Psychophysiology, 44, 620-626. doi:10.1111/j.1469-8986.2007.00524.x

Ghisolfi, E. S., Heldt, E., Zanardo, A. P., Strimitzer, I. M., Jr., Prokopiuk, A. S., Becker, J., ET AL. (2006). P50 sensory gating in panic disorder. Journal of Psychiatry Research, 40, 535-540. doi:10.1016/j.jpsychires.2006.02.006

Gooding, D. C., Braun, J. G., \& Studer, J. A. (2006). Attentional network task performance in patients with schizophrenia-spectrum disorders: Evidence of a specific deficit. Schizophrenia Research, 88, 169-178. doi:10.1016/j.schres.2006.07.009

Gottesman, I. I., \& Gould, T. D. (2003). The endophenotype concept in psychiatry: Etymology and strategic intentions. American Journal of Psychiatry, 160, 636-645. doi:10.1176/appi.ajp.160.4.636

Grunwald, T., Boutros, N. N., Pezer, N., von Oertzen, J., FernánDEZ, G., Schaller, C., \& Elger, C. E. (2003). Neuronal substrates of sensory gating within the human brain. Biological Psychiatry, 53, 511-519.

Guterman, Y., \& Josiassen, R. C. (1994). Sensory gating deviance in schizophrenia in the context of task related effects. International Journal of Psychophysiology, 18, 1-12.

Guterman, Y., Josiassen, R. C., \& Bashore, T. R., Jr. (1992). Attentional influence on the P50 component of the auditory eventrelated brain potential. International Journal of Psychophysiology, 12, 197-209.

Heal, L., \& Johnson, J. T., JR. (1970). Inhibition deficits in retardate learning and attention. In N. Ellis, P. Siegel, \& I. Bjorgen (Eds.), International review of research in mental retardation (pp. 107-149). New York: Academic Press.

Healey, M. K., Campbell, K. L., \& Hasher, L. (2008). Cognitive aging and increased distractibility: Costs and potential benefits. Progress in Brain Research, 169, 353-363.

Jerger, K., Biggins, C., \& Fein, G. (1992). P50 suppression is not affected by attentional manipulations. Biological Psychiatry, 31, 365-377.

Jessen, F., Kucharski, C., Fries, T., Papassotiropoulos, A., HoeNIG, K., MaIer, W., \& Heun, R. (2001). Sensory gating deficit expressed by a disturbed suppression of the P50 event-related potential in patients with Alzheimer's disease. American Journal of Psychiatry, 158, 1319-1321. doi:10.1176/appi.ajp.158.8.1319

Kisley, M. A., Noecker, T. L., \& Guinther, P. M. (2004). Comparison of sensory gating to mismatch negativity and self-reported perceptual phenomena in healthy adults. Psychophysiology, 41, 604-621.

Knight, R. T., Staines, W. R., Swick, D., \& ChaO, L. L. (1999). Prefrontal cortex regulates inhibition and excitation in distributed neural networks. Acta Psychologica, 101, 159-178.

Korzyukov, O., Pflieger, M. E., Wagner, M., Bowyer, S. M., RosBURG, T., SUNDARESAN, K., ET AL. (2007). Generators of the intracranial P50 response in auditory sensory gating. NeuroImage, 35, 814-826.

Kramer, A. F., Humphrey, D. G., Larish, J. F., Logan, G. D., \& Strayer, D. L. (1994). Aging and inhibition: Beyond a unitary view of inhibitory processing in attention. Psychology \& Aging, 9, 491-512. doi:10.1037/0882-7974.9.4.491

Kurthen, M., Trautner, P., Rosburg, T., Grunwald, T., Dietl, T., KüHN, K., ET AL. (2007). Toward a functional topography of sensory gating areas: Invasive P50 recording and electrical stimulation mapping in epilepsy surgery candidates. Psychiatry Research, 155, 121-133.

Làdavas, E., Petronio, A., \& Umiltà, C. (1990). The deployment of visual attention in the intact field of hemineglect patients. Cortex, 26, 307-317.

Lavie, N. (1995). Perceptual load as a necessary condition for selective attention. Journal of Experimental Psychology: Human Perception \& Performance, 21, 451-468. doi:10.1037/0096-1523.21.3.451

Lavie, N., Hirst, A., De Fockert, J. W., \& Viding, E. (2004). Load theory of selective attention and cognitive control. Journal of Experimental Psychology: General, 133, 339-354. doi:10.1037/0096 $-3445.133 .3 .339$

LudBrook, J. (1998). Multiple comparison procedures updated. Clinical \& Experimental Pharmacology \& Physiology, 25, 1032-1037.

Lustig, C., Hasher, L., \& Zacks, R. T. (2007). Inhibitory deficit theory: Recent developments in a "new view." In D. S. Gorfein \& C. M. MacLeod (Eds.), Inhibition in cognition (pp. 145-162). Washington, DC: American Psychological Association. doi:10.1037/11587-008

Luu, P., \& Ferree, T. (2005). Determination of the Geodesic Sensor Nets' average electrode positions and their 10-10 international equivalents. (Tech. Note). Eugene, OR: Electrical Geodesics, Inc.

MacLeod, C. M., \& Dunbar, K. (1988). Training and Stroop-like interference: Evidence for a continuum of automaticity. Journal of Experimental Psychology: Learning, Memory, \& Cognition, 14, 126-135. doi:10.1037/0278-7393.14.1.126

MARLOW-O'ConNer, M. A. (2005). fMRI indices of frontal activation associated with sensory gating deficits in schizophrenia. Dissertation Abstracts International, 66B, 3417.

McGhie, A., \& Chapman, J. (1961). Disorders of attention and perception in early schizophrenia. British Journal of Medical Psychology, 34, 103-116. 
Nagamoto, H. T., Adler, L. E., Hea, R. A., Griffith, J. M., McRae, K. A., \& FreEdMAN, R. (1996). Gating of auditory P50 in schizophrenics: Unique effects of clozapine. Biological Psychiatry, 40, 181-188.

Nagamoto, H. T., Adler, L. E., Waldo, M. C., Griffith, J., \& FreedMAN, R. (1991). Gating of auditory response in schizophrenics and normal controls. Effects of recording site and stimulation interval on the P50 wave. Schizophrenia Research, 4, 31-40.

NeE, D. E., WAGER, T. D., \& Jonides, J. (2007). Interference resolution: Insights from a meta-analysis of neuroimaging tasks. Cognitive, Affective, \& Behavioral Neuroscience, 7, 1-17.

NeILl, W. T. (1977). Inhibition and facilitation processes in selective attention. Journal of Experimental Psychology: Human Perception \& Performance, 3, 444-450. doi:10.1037/0096-1523.3.3.444

Olincy, A., Ross, R. G., Harris, J. G., Young, D. A., McAndrews, M. A., CAWThra, E., ET AL. (2000). The P50 auditory event-related potential in adult attention-deficit disorder: Comparison with schizophrenia. Biological Psychiatry, 47, 969-977.

Patterson, J. V., Hetrick, W. P., Boutros, N. N., Jin, Y., Sandman, C., STERN, H., ET AL. (2008). P50 sensory gating ratios in schizophrenics and controls: A review and data analysis. Psychiatry Research, 158, 226-247.

Persons, J. B. (1986). The advantages of studying psychological phenomena rather than psychiatric diagnoses. American Psychologist, 41, 1252-1260.

PICARD, N., \& STRICK, P. L. (2001). Imaging the premotor areas. Current Opinion in Neurobiology, 11, 663-672.

Picton, T. W., Stuss, D. T., Alexander, M. P., Shallice, T., Binns, M. A., \& Gillingham, S. (2007). Effects of focal frontal lesions on response inhibition. Cerebral Cortex, 17, 826-838.

Potter, D., Summerfelt, A., Gold, J., \& Buchanan, R. W. (2006). Review of clinical correlates of P50 sensory gating abnormalities in patients with schizophrenia. Schizophrenia Bulletin, 32, 692-700.

SAlthouse, T. A. (1996). The processing-speed theory of adult age differences in cognition. Psychological Review, 103, 403-428.

Schwartz, S., Vuilleumier, P., Hutton, C., Maravita, A., Dolan, R. J., \& Driver, J. (2005). Attentional load and sensory competition in human vision: Modulation of fMRI responses by load at fixation during task-irrelevant stimulation in the peripheral visual field. Cerebral Cortex, 15, 770-786.

Smith, D. A., Boutros, N. N., \& Schwarzkopf, S. B. (1994). Reliability of P50 auditory event-related potential indices of sensory gating. Psychophysiology, 31, 495-502. doi:10.1111/j.1469-8986.1994 .tb01053.x

Steel, C., Haworth, E. J., Peters, E., Hemsley, D. R., Sharma, T., GrAY, J. A., ET AL. (2001). Neuroimaging correlates of negative priming. NeuroReport, 12, 3619-3624.

STROOP, J. R. (1935). Studies of interference in serial verbal reactions. Journal of Experimental Psychology, 18, 643-662. doi:10.1037/ h0054651

Talay-Ongan, A., \& Wood, K. (2000). Unusual sensory sensitivities in autism: A possible crossroads. International Journal of Disability Development \& Education, 47, 201-212. doi:10.1080/713671112

TIPPER, S. P. (1985). The negative priming effect: Inhibition priming by ignored objects. Quarterly Journal of Experimental Psychology, 37A, 571-590.

Tregellas, J. R., Davalos, D. B., Rojas, D. C., Waldo, M. C., GibSON, L., WYLIE, K., ET AL. (2007). Increased hemodynamic response in the hippocampus, thalamus and prefrontal cortex during abnormal sensory gating in schizophrenia. Schizophrenia Research, 92, 262-272.

Uc, E. Y., Skinner, R. D., Rodnitzky, R. L., \& Garcia-Rill, E. (2003). The midlatency auditory evoked potential P50 is abnormal in Huntington's disease. Journal of the Neurological Sciences, 212, 1-5.

Venables, P. H. (1964). Input dysfunction in schizophrenia. Progress in Experimental Personality Research, 72, 1-47.

Waldo, M. C., Cawthra, E., Adler, L. E., Dubester, S., Staun-
TON, M., NaGamoto, H., ET AL. (1994). Auditory sensory gating, hippocampal volume, and catecholamine metabolism in schizophrenics and their siblings. Schizophrenia Research, 12, 93-106.

Weisbrod, M., Kiefer, M., MarzinziK, F., \& Spitzer, M. (2000). Executive control is disturbed in schizophrenia: Evidence from event-related potentials in a go/no-go task. Biological Psychiatry, 47, 51-60.

Weisser, R., Weisbrod, M., Roehrig, M., RupP, A., Schroeder, J., \& ScherG, M. (2001). Is frontal lobe involved in the generation of the auditory evoked P50? NeuroReport, 12, 3303-3307.

Wright, C. I., Keuthen, N. J., Savage, C. R., Martis, B., WilLIAMS, D., Wedig, M., ET AL. (2006). Brain correlates of negative and positive visuospatial priming in adults. NeuroImage, 30, 983-991.

\section{NOTES}

1. References to sensory gating and P50 gating in this and subsequent sections refer to that of the auditory type, as is defined here, unless otherwise specified.

2. $40 \mathrm{k} \Omega$ is the manufacturer-recommended standard for this system.

3. Wherever applicable, P50 analysis parameters were taken from Kisley et al. (2004).

4. Neither N100 amplitude nor gating (using the $T / C$ ratio) was significantly related to any of the cognitive inhibition measures.

5. Some researchers have found the $T / C$ ratio quantification of P50 suppression to have poor reliability (Fuerst, Gallinat, \& Boutros, 2007), and a difference score $(C-T)$ has been proposed as an alternative quantification because of its better test-retest reliability relative to the $T / C$ ratio (Smith, Boutros, \& Schwarzkopf, 1994). Although difference scores are used far less frequently than the $T / C$ ratio, we conducted these analyses given this measure's enhanced reliability. Although our results were in the expected direction, the effects did not reach significance [P50 gating and Stroop interference, $r(21)=.337$, n.s.; P50 gating and go/no-go stopping error, $r(23)=-.215$, n.s.; P50 gating and Eriksen flanker interference, $r(21)=-.077, \mathrm{n} . \mathrm{s}$.]. Note that for the difference scores, a higher value reflects better gating, and for the $T / C$ ratio, a higher value reflects worse gating. (This explains the reversal in direction of our correlations.)

6. We performed a correction for the above multiple tests (we adjusted our alpha level to reduce the risk of Type I error). We used the Holm-Bonferroni step-down method because it controls Type I error rate comparably to the well-known Bonferroni correction, yet offers better power (Ludbrook, 1998). The adjusted $p$ values for the comparisons among our cognitive inhibition measures and auditory P50 gating are as follows: P50 gating and Stroop interference, $p=$ .042 (from .014); P50 gating and go/no-go stopping error, $p=.046$ (from .023); and P50 gating and Eriksen flanker interference, $p=$ 1.91 (from .953; note that a Bonferroni $p$ can exceed 1 -Ludbrook, 1998). The adjusted $p$ values indicate that our effects withstood correction for multiple comparisons.

7. Two participants' scores strongly diverged from the first to the second half of the go/no-go task. When these participants were removed, the split-half reliability was highly significant $[r(21)=.716$, $p<.001$, Cronbach's $\alpha=.817$ ].

8 . The same pattern of results was obtained when we limited the correlational analysis of P50 suppression and Stroop $[r(21)=-.523$, $p=.010]$ and of P50 suppression and go/no-go $[r(21)=-.455$, $p=.029]$ to those participants who had data for all three measures $(n=23)$.

(Manuscript received September 30, 2008; revision accepted for publication July 1,2009 .) 